\title{
Headache and Acute Illness in Children
}

\author{
Raymond S. Kandt, MD; Robert M. Levine, MD
}

\begin{abstract}
Thirty-seven children with headaches who were seen in a walk-in clinic were matched to 37 headache-free controls. Thirty percent of the headache group and $11 \%$ of the headache-free control group had a body temperature above $38^{\circ} \mathrm{C}(p<0.05)$. Nonrhythmic pain was more commonly associated with fever than was rhythmic pain $(p<0.05)$. Of 34 headache subjects who completed questionnaires, those with more intense headaches reported a greater number of headache-exacerbating factors $(p<0.01)$. Bilateral headaches were more painful than unilateral headaches, and in two thirds of the subjects, the intensity of pain paralleled the course of the underlying illness. A family history of migraine was more common in the headache group as compared to the headache-free control group $(p<0.05)$. Headaches associated with acute illnesses may be a precursor to later migraine. (J Child Neurol 1987;2:22-27)
\end{abstract}

$\mathrm{H}^{\mathrm{e}}$ eadaches in children and adolescents are often perceived as a relatively unimportant part of illness. Little is known about the relationship between headaches and acute illness in children. The purposes of this prospective study were to develop a detailed clinical description of illness-associated headaches; to determine the relationships among fever, acute illness, and headache; to describe the relationship of headache to the course of the underlying illness, and to see if a relationship exists with childhood migraine.

\section{Methodology}

Parents of children and adolescents from 3 to 15 years of age presenting to the pediatric walk-in clinic were asked by the registrar if headache was one of the child's symptoms. If the parent or child answered "yes," the child was entered into the study. Children requiring immediate medical attention were excluded. Subjects with headaches were matched to headache-free controls who visited the clinic for acute

Received July 18, 1985. Received revised Oct 8, 1985. Accepted for publication Oct 15, 1985.

From the departments of pediatrics and neurology, University of Michigan Medical Center (Dr Kandt) and the University of Michigan Medical School (Dr Levine), Ann Arbor, MI

Address correspondence to Raymond S. Kandt, MD, Pediatric Neurology, PO Box 3533, Duke University Medical Center, Durham, NC 27710. illnesses. Matching was done by age, sex, and date of visit. Vital signs (temperature, pulse rate, respiratory rate, and blood pressure) and final diagnoses were obtained by a later chart review. A questionnaire, to be completed in the examining or waiting room, examined both the temporal course and perceived severity of the illness and the headache, and provided information on the features of the headacheintensity, duration, quality, location, and exacerbating and relieving factors. Intensity was ranked as mild, medium, severe, or "the worst pain in my life." Attempts were made to create a short and easily understandable questionnaire. Possible responses were listed next to the questions, and front- and sideview drawings of a head were included for the patient to mark the location or locations of the headache. The information obtained was confirmed for accuracy in follow-up telephone interviews.

Because the head pain of both migraine and acute illness is thought to arise, in part, from cerebral vasodilatation, we felt that finding a similarity between the clinical features of the acute illnessassociated headaches and migraine would not contribute to showing a relationship between the two entities. By contrast, if we found that our headache subjects and more non-headache features that have been associated with migraine (eg, sleep disorders and a family history of migraine) than the control subjects, an association between the headaches of acute illness and migraine would be suggested. 
Therefore, standardized follow-up telephone interviews obtained information from headache subjects and headache-free controls on sleep disorders, family history of migraine headaches, and recurrence of headaches in the two weeks since the visit. Four weeks after the visit, further information was obtained by telephone regarding the subjects' general health (ie, "How has [John] been feeling since I called you last?") and the occurrence of headaches since the follow-up two weeks previously.

The chi square test (uncorrected) or the paired $t$ test was used to assess statistical significance.

\section{Results}

Of 46 patients presenting with acute illnesses and headaches, 21 were male and 25 female (no significant difference). Ages ranged from 3 to 15 years, with a mean of 9 years. There was no preponderance of males or females in any age group.

Vital signs were available for 37 subjects with headaches. These subjects were matched to 37 headache-free controls by sex, age, and date of visit. Diagnoses are listed in Table 1 and categorized as infectious or noninfectious. Pharyngitis was the most common diagnosis in both the headache and headache-free groups. All the children in the noninfectious category were afebrile. Headaches were a secondary complaint in all but four of the children in the headache group. Although the difference between the mean temperatures of the groups was statistically significant $(p<0.05)$, the mean temperature of the headache group $\left(37.6^{\circ} \mathrm{C}\right)$ did not appear much higher than the mean temperature of the control group $\left(37.1^{\circ} \mathrm{C}\right)$. However, $30 \%$ of the headache group, but only $11 \%$ of the headache-free control group, had a body temperature above $38^{\circ} \mathrm{C}(p<$ 0.05 ). Dehydration was not specifically noted in any of the cases. Furthermore, there was no statistical difference between groups for pulse rate, respiratory rate, or blood pressure. Neither meningeal signs nor alteration of consciousness suggestive of encephalitis was recorded for any patient.

\section{Telephone Follow-up}

Twenty-nine headache patients and 27 controls agreed to telephone follow-up two weeks after their clinic visits. In the two weeks following the clinic visits, 18 of 29 headache patients (62\%) and eight of 27 controls $(30 \%)$ had one or more days of headache $(p<0.05)$. At telephone follow-up one month after the clinic visit, five of 27 headache patients $(18.5 \%)$ and one of 22 control subjects $(4.5 \%)$ had experienced
TABLE 1

Diagnoses of 37 Headache Subjects Matched with 37

Headache-Free Controls

\begin{tabular}{|c|c|c|}
\hline & $\begin{array}{c}\text { Headache } \\
\text { Group }\end{array}$ & $\begin{array}{c}\text { Control } \\
\text { Group }\end{array}$ \\
\hline \multicolumn{3}{|l|}{ Infectious disorders } \\
\hline Pharyngitis & 8 & 7 \\
\hline Gastroenteritis & 1 & 4 \\
\hline $\begin{array}{l}\text { Lower respiratory infections } \\
\text { (bronchitis and pneumonia) }\end{array}$ & 1 & 3 \\
\hline Upper respiratory infections & 2 & 1 \\
\hline Otitis media & 1 & 2 \\
\hline Urinary tract infection & 2 & 2 \\
\hline Nonspecific infection & 2 & 0 \\
\hline Lymphadenopathy & 2 & 1 \\
\hline Chicken pox & 1 & 1 \\
\hline Influenza & 1 & 0 \\
\hline Fever, etiology unknown & 1 & 0 \\
\hline Infected toe & 0 & 1 \\
\hline Scabies & 0 & 1 \\
\hline Conjunctivitis & 0 & 1 \\
\hline Subtotals & 22 & 24 \\
\hline \multicolumn{3}{|l|}{ Noninfectious disorders } \\
\hline Asthma & 3 & 3 \\
\hline Nonhead injuries (minor) & 2 & 3 \\
\hline Head trauma, mild & 1 & 0 \\
\hline Headache & 4 & 0 \\
\hline Allergic reactions & 1 & 2 \\
\hline Insect bites & 0 & 2 \\
\hline Seizures & 1 & 1 \\
\hline Dyspnea & 1 & 0 \\
\hline Epididymitis $v$ hematoma & 1 & 0 \\
\hline Swollen feet & 0 & 1 \\
\hline Subtotals & 14 & 12 \\
\hline No diagnosis & 1 & 1 \\
\hline TOTAL & 37 & 37 \\
\hline
\end{tabular}

one or more days of headache during weeks 3 and 4 after the clinic visit. This difference was not statistically significant $(0.1<p<0.2)$.

By four weeks, $85.0 \%$ of the headache group and $81.8 \%$ of the headache-free control group were fully recovered. None of the children who were unwell complained of major discomfort or symptoms suggesting a serious illness.

Thirteen of 29 headache patients $(44.8 \%)$ and five of 27 controls $(18.5 \%)$ had a family history of migraine $(p<0.05)$.

No significant increase was found in sleep disorders in the headache group (see Table 2). Disorders examined included sleepwalking, talking in sleep, nocturnal enuresis, difficulty going to sleep, fitful or uneasy sleep, and rocking head or body while asleep. Nocturnal enuresis was four times as frequent in the headache-free control group as in the headache group, a statistically significant difference $(p<0.01)$. The majority of subjects in the headache and head- 
TABLE 2

Sleep Disorders in Headache Subjects $v$ Headache-Free Controls

\begin{tabular}{|c|c|c|c|}
\hline & $\begin{array}{c}\text { Headache } \\
\text { Subjects } \\
(\mathrm{N}=29)\end{array}$ & $\begin{array}{l}\text { Headache- } \\
\text { free Con- } \\
\text { trols } \\
(\mathrm{N}=27)\end{array}$ & $p$ Value \\
\hline Sleepwalking & 8 & 3 & NS \\
\hline Talking in Sleep & 19 & 19 & NS \\
\hline Bed-wetting & 3 & 12 & $<0.01$ \\
\hline Difficulty getting to sleep & 6 & 5 & NS \\
\hline Fitful sleep & 9 & 6 & NS \\
\hline $\begin{array}{l}\text { Rocking head or body } \\
\text { while sleeping }\end{array}$ & 2 & 1 & NS \\
\hline Any of above & 23 & 22 & NS \\
\hline
\end{tabular}

ache-free control groups reported at least one of the sleep disorders studied.

\section{Headache Characteristics}

Questionnaires were completed for 34 subjects in the headache group, 16 males and 18 females. In terms of intensity, two subjects ranked their headaches as mild, 14 as medium, 16 as severe and two as "the worst pain in my life." Twenty-five subjects $(73.5 \%)$ had bilateral headaches at onset, and nine $(26.5 \%)$ had unilateral headaches at onset. In two cases, the headache location at the time of the questionnaire was different from the location at onset. Of these two cases, headache in one subject changed from bilateral to unilateral, and one changed from unilateral to bilateral. The most common pain quality was pounding or throbbing, which was experienced by 14 subjects $(41.2 \%)$. Ten subjects $(29.4 \%)$ reported their headaches as purely pounding or throbbing, while four other subjects reported pounding or throbbing as a component of the headache pain. Steady aching was experienced by 11 subjects $(32.4 \%$ )-as the sole pain in seven subjects, and in combination for four others. When pain quality was divided into two basic groups, rhythmic pain (pounding or throbbing, jabbing, stabbing, bursting, exploding) and nonrhythmic pain (steady ache, pressure, burning), it was found that 19 subjects $(55.9 \%)$ had a component of rhythmic pain, and 11 subjects $(32.4 \%)$ experienced only nonrhythmic pain. Four subjects did not specify the pain quality. Seventeen subjects $(50 \%)$ had experienced headache for a day or less at the time of the visit, and 15 subjects $(44.1 \%)$ had had headaches for several days to more than a week prior to their visits.

The 34 subjects reported 12 different exacerbating factors for their headaches and seven different relieving factors. Nineteen subjects reported more than one exacerbating factor. The most common exacerbating factor was a movement of some type (see Table 3). Movement was an exacerbating factor for 28 subjects $(82.4 \%)$, and was the only exacerbating factor for 14 subjects $(41.2 \%)$. Primary sensory stimuli (light, noise, darkness) were exacerbating factors for a total of 16 subjects $(47.1 \%)$, but were combined with some type of movement in 13 of these subjects $(38.2 \%)$. They were the sole exacerbating factors in only three subjects $(8.8 \%)$. Light and noise were paired as exacerbating factors in seven of the 16 subjects for whom these were exacerbating factors, but were unassociated with the other sensory stimulus in nine of the 16 subjects. Only one subject reported fever as an exacerbating factor.

Nineteen subjects reported more than one relieving factor. The most common relieving factor was lying down (see Table 4). Lying down was a relieving

TABLE 3

Exacerbating Factors of 34 Headache Subjects

\begin{tabular}{lcc}
\hline \multicolumn{1}{c}{ Factor } & $\begin{array}{c}\text { No. of } \\
\text { Subjects }^{*}\end{array}$ & Percent \\
\hline Type of Movement & & \\
Standing & 18 & 52.9 \\
Head movement & 12 & 35.3 \\
Coughing & 11 & 32.4 \\
Vomiting & 2 & 5.9 \\
Lying down & 2 & 5.9 \\
Swallowing & 1 & 2.9 \\
Walking & 1 & 2.9 \\
Sitting & 1 & 2.9 \\
Primary Sensory Stimulus & 13 & 38.2 \\
Noise & 10 & 29.4 \\
Light & 1 & 2.9 \\
Darkness & & \\
Other & 1 & 2.9 \\
Fever & & \\
\hline
\end{tabular}

* Several subjects reported more than one exacerbating factor.

TABLE 4

Relieving Factors of 34 Headache Subjects

\begin{tabular}{lcc}
\hline \multicolumn{1}{c}{ Factor } & $\begin{array}{c}\text { No. of } \\
\text { Subjects }\end{array}$ & Percent \\
\hline Lying down & 24 & 70.6 \\
Quiet & 13 & 38.2 \\
Darkness & 8 & 23.5 \\
Medication & & \\
$\quad$ Acetaminophen & 8 & 23.5 \\
Aspirin & 7 & 20.6 \\
Codeine & 1 & 2.9 \\
Massage & 3 & 8.8 \\
Vomiting & 2 & 5.9 \\
Ice pack & 1 & 2.9 \\
\hline
\end{tabular}

* Several subjects reported more than one relieving factor. 
factor for 24 subjects $(70.6 \%)$, and was the only relieving factor noted by seven subjects. Quiet and/or darkness were relieving factors in 15 subjects $(44.1 \%)$, but were combined with lying down in all but four subjects. Lying down, darkness, and quiet, either alone or in combination with each other or other factors, helped relieve the headaches of 28 subjects $(82.4 \%)$. Fifteen subjects $(44.1 \%)$ reported medication as a relieving factor, but only three reported medication as a relieving factor, but only three reported medication as the sole relieving factor. One subject noted vomiting as the sole relieving factor.

\section{Interrelationships}

Headache location and intensity were not related to body temperature. Six of ten subjects $(60 \%)$ with solely nonrhythmic pain had a fever (body temperature was not available for the 11th subject in this group), but only three of 19 subjects $(15.8 \%)$ with rhythmic pain had a fever $(p<0.05)$. Two of the ten subjects with only pounding or throbbing pain (a subgroup of rhythmic pain) had fever.

Of the 34 headache subjects who completed questionnaires, nine subjects $(26.5 \%)$ reported unilateral headaches. Over three fourths $(78 \%)$ of subjects with unilateral headaches reported them as mild or medium in intensity, as compared to two thirds $(64 \%)$ with bilateral headaches who reported them as severe or "the worst pain in my life" ( $p$ $<0.05$ ). Most males $(88 \%)$ reported bilateral headaches, while females were more evenly split with $39 \%$ reporting unilateral and $61 \%$ reporting bilateral headaches. This trend, however, did not reach statistical significance $(0.05<p<0.1)$.

Almost every subject reported that the headache had been continuous from the onset of the illness, or that headaches had recurred since the onset of the illness. Subjects rated the illness and the headache independently as improving, staying the same, or worsening. In two thirds of subjects, the course of the headaches paralleled the course of the illness. Those who reported that their illnesses were worsening, for example, tended to report that their headaches were worsening as well.

Headache intensity was compared with the number of factors listed as exacerbating the headache (see Figure 1). As headache intensity increased, so did the number of factors that exacerbated the headache $(p<0.01)$. None of the following factors showed a significant relationship to the intensity of headache pain: headache location (ie, frontal, temporal, occipital, orbital, or retro-orbital); pain quality;

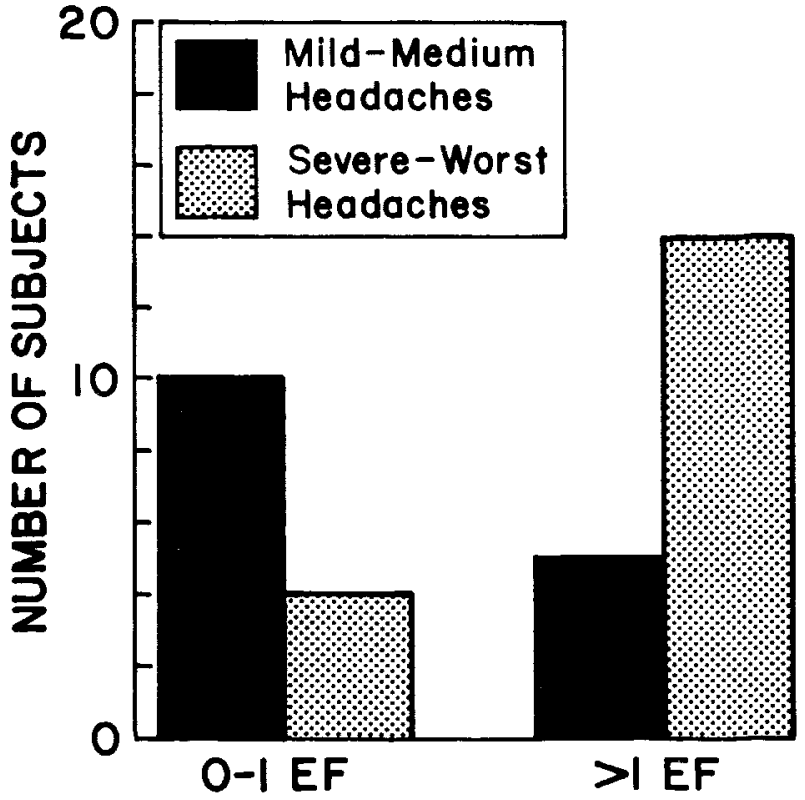

FIGURE 1

Headache intensity related to number of exacerbating factors (EF).

infectious or noninfectious etiology; age of the subject; number of relieving factors; or duration of headache prior to clinic visit. There was also no relationship between the various exacerbating and relieving factors and the quality of headache pain (pounding or throbbing, pressure, steady ache).

\section{Discussion}

Although fever is considered a common cause of headache, ${ }^{1}$ this relationship has not been systematically studied in children. In fact, data relating to acute illness-associated headaches in children are not available. ${ }^{2}$ Our controlled study demonstrates that children who have a headache at the time they are seen in the clinic are more likely to have a fever than those children who do not have a headache. Although the mean temperature of the control and headache groups differed only slightly $\left(37.1^{\circ} \mathrm{C} v 37.6^{\circ} \mathrm{C}\right.$ respectively $-p<0.05$ ), there were almost three times as many children in the headache group who had fever (temperature above $38^{\circ} \mathrm{C}$ ). However, the height of the fever did not correlate with the location of the headache or intensity of the pain. The common concept that headache associated with fever is pounding or throbbing ${ }^{3}$ was not confirmed by this study. In fact, the opposite situation occurred. Fever (temperature greater than $38^{\circ} \mathrm{C}$ ) was present in $60 \%$ 
of the subjects whose pain was solely nonrhythmic (steady ache, pressure, burning), but fever was present in only $15.8 \%$ of subjects who had a component of rhythmic pain (pounding, jabbing, stabbing, exploding $-p<0.05$ ). Additionally, there was no relationship between the intensity and the quality of the pain (pounding or throbbing, pressure or steady ache). Aggravation of the headache by movement was common in our subjects and is consistent with prior observations. ${ }^{1}$

Associated illnesses in the headache and headache-free control groups were similar; pharyngitis was the most common diagnosis in both groups. Many of the children in this study who had infectious illnesses were afebrile. This is not surprising, as it is well-known that common pediatric infections such as pharyngitis, otitis media, and gastroenteritis may occur in the absence of fever. ${ }^{4,5}$ However, those children who had headaches in association with their illnesses were more likely to have fever. Whether a subject's headache was due to the fever, or whether both the fever and the headache resulted from a more severe primary illness, cannot be determined by this study. On telephone follow-up four weeks after the clinic visit, the acute illness that occasioned the initial visit had resolved in most children of both groups. When asked how their child had been feeling, none of the parents reported symptoms suggestive of meningitis, brain abscess, increased intracranial pressure, brain hemorrhage, or focal neurological problems. The headaches experienced by these children were of variable quality and intensity. Although no serious illnesses occurred in the subjects of our study, parents felt that the severity of the headaches paralleled the severity of the illness. Therefore, while the headache characteristics do not seem reliable as a gauge for the severity of a child's illness, a worsening headache may be useful as an indicator for a worsening illness.

Both migraine headaches and headaches associated with acute illnesses are thought to have a vascular basis, and therefore a throbbing, rhythmic quality to the pain. Overall, the majority of children $(55.9 \%)$ in this study who had headaches and acute illnesses had headaches with a rhythmic quality to the pain. Considering the possibility of this result when designing the study, we also looked at features other than the quality of pain in our patients (eg, family history of migraine, presence of sleep disorders, and recurrence of headaches) to see if a relationship exists between migraine and the headaches of acute illness. Such a relationship is supported by the frequent occurrence of a family history of migraine in the headache group $(44.8 \%)$ as compared to the headache-free control group (18.5\%) $(p<0.05)$. The similarity of this family history data to that obtained by other studies is striking. Raskin and Appenzeller ${ }^{7}$ in their review, found that $50 \%$ to $60 \%$ of subjects with migraine headaches had a parent with migrainous headaches, but only $10 \%$ to $20 \%$ of subjects who did not have headaches had a parent with migrainous headaches. In the general population, severe headaches are a common problem. It has been estimated that over $40 \%$ of North Americans have suffered severe headaches at some time during their lives. ${ }^{8}$ The annual prevalence of migraine is probably higher than $20 \%$ to $25 \% .{ }^{7}$ Viewed in the context of these studies, the large numbers of children in our study who had a family history of migraine headaches is not at all surprising. However, a history of sleep disorders, which has been reported as more frequent in children with migraine than those without, ${ }^{9,10}$ occurred with almost the same frequency in both groups (see Table 2). Surprisingly, nocturnal enuresis was more common in the control group $(p<0.01)$. When telephoned two weeks after the clinic visit, significantly more of the headache patients were still experiencing headaches $(p<0.05)$, but by four weeks after the visit, there was no significant difference between groups. This duration of follow-up was admittedly too short to judge whether or not the headache group was more or less prone to recurrent headaches than the headache-free control group. In view of the strong family history of migraine in our headache group as compared to the headache-free control group, it is possible that children with headaches as a component of an acute illness will later develop recurrent headaches of the migraine type. Further follow-up of such children will be necessary to see if this occurs.

The average subject with illness-associated headaches experienced bilateral, medium to severe headaches with rhythmic pain exacerbated by movement and relieved by lying down. Unilateral headaches, in addition to occurring less commonly $(26.5 \%$ of subjects), were also less intense than the bilateral headaches. The unilateral headaches were more often mild to medium in intensity, whereas bilateral headaches were more often severe to the worst pain ever experienced $(p<0.05)$.

Subjects who noted multiple exacerbating factors for their headaches tended to have more intensely painful headaches (see Figure 1). There are several possible explanations for this relationship. As headaches became more intense, the sensitivity to environmental influences (movements, sensory 
stimuli) may have increased, and influences that were heretofore benign became irritating, leading to further worsening of the headache. Alternatively, the subjects with the more severe headaches may have been more active and likely to be involved in noisy activities illuminated by bright light. That is, their personality types or the exigencies of their lifestyles may have predisposed them to situations that worsen headaches. It may be that subjects who noted fewer exacerbating factors and milder headaches controlled their environment so that they experienced less of the stimuli that exacerbate headaches. States of moderate anxiety increase the perception of pain. ${ }^{11}$ Anxiety may also decrease the threshold at which environmental factors exacerbate the pain, resulting in a concomitance of more intense pain and a larger number of exacerbating factors. The headache subjects in general tended to perceive the course of their illnesses (eg, improving, staying the same, worsening) as paralleling the course of their headaches. Rather than the two processes being independent, it is possible that anxiety associated with a worsening illness promoted the perception of pain. Migraine patients are known to have headaches associated with stress. ${ }^{12}$ One postulate suggests that migraine sufferers are more vulnerable to headaches at such times because of the alternation of internal "milieu."13 A similar explanation is possible for the headaches associated with acute illnesses in children, in that a child's routine is increasingly disrupted as the illness becomes more severe. The simplest explanation, however, for the positive association between the number of exacerbating factors and headache intensity is that some individuals have a predisposition to headache with a lowered threshold to multiple triggering and exacerbating factors.

Theoretically, fever may have a therapeutic value. ${ }^{14}$ However, the association between headache and fever demonstrated in this study supports the symptomatic use of an analgesic/antipyretic agent when headache is part of a child's illness. Further- more, the other mainstay of nonspecific treatment, ie, rest in a quiet environment without bright illumination, may decrease morbidity partly by its beneficial effect on any attendant headache. Not only should the complaint of headache be taken seriously because it may respond to symptomatic treatment, but a worsening headache may signal a worsening primary illness.

\section{References}

1. Dalessio DJ: Toxic vascular headache, in Dalessio DJ (ed): Wolff's Headache and Other Head Pain, ed 4. New York, Oxford University Press, 1980, pp 198-219.

2. Rothner AD: Diagnosis and management of headache in children and adolescents. Neurol Clin 1983;1:511-526.

3. Saper JR: Headache Disorders. Current Concepts and Treatment Strategies. Boston, John Wright PSG Inc., 1983, p 158.

4. Behrman RE: Acute pharyngitis, in Behrman RE, Vaughn VC, Nelson WE (eds): Nelson Textbook of Pediatrics, ed 12. Philadelphia, WB Saunders, 1983, p 1015.

5. Bluestone CD: The ear, in Behrman RE, Vaughn VC, Nelson WE (eds): Nelson Textbook of Pediatrics, ed 12. Philadelphia, WB Saunders, 1983, p 1026.

6. Krugman S, Katz SL: Infectious Diseases of Children. St. Louis, CV Mosby, 1981, pp 63, 236.

7. Raskin NH, Appenzeller O: Headache. Major Problems in Internal Medicine. Philadelphia, WB Saunders, 1980, vol 19, pp 21-32.

8. Ziegler DK, Hassanein RS, Couch JR: Characteristics of life headache histories in a nonclinic population. Neurology 1977;27:265-269.

9. Barabas G, Ferrari M, Matthews WS: Childhood migraine and somnambulism. Neurology 1983;33:948-949.

10. Bille B: Migraine in school children. Acta Paediatr 1962; 51(suppl 136):1-151.

11. Merskey H: Pain and emotion: Their correlation in headache, in Critchley M, Friedman AP, Gorini S, Sicuteri F (eds): Headache: Physiopathological and Clinical Concepts. In Advances in Neurology. New York, Raven Press, 1982, vol 33, pp 135-143.

12. Friedman AP: Headache, in Baker AB, Baker LH (eds): Clinical Neurology. Philadelphia, Harper \& Row, 1983, ch 13, pp 8-9, 21- 22.

13. Diamond S, Medina JL: Review article: current thoughts on migraine. Headache 1980;20:208-212.

14. Stern RC: Pathophysiologic basis for symptomatic treatment of fever. Pediatrics 1977;59:92-98. 